\title{
Influence of the shape and size of a dummyhead upon Thévenin acoustic impedance and Thévenin pressure
}

\author{
Kimitoshi Fukudome and Masaki Yamada ${ }^{\dagger}$ \\ Department of Acoustic Design, Kyushu Institute of Design, \\ 4-9-1, Shiobaru Minami-ku Fukuoka, 815 Japan
}

(Received 6 February 1988)

\begin{abstract}
The influence of shape and size of a dummyhead upon the sound reaching the ear is discussed. The Thévenin acoustic impedance and Thévenin pressure, which have been adopted as acoustical parameters for evaluating the effect of individually different shapes and sizes of the human head (K. Fukudome, Proc. 10th Int. Congr. Acoust. (Sydney) 1980), are numerically examined by using two types of model heads. The shape and size of the model heads, respectively a sphere and an oblate spheroid, were changed according to head circumference and the cephalic index of the somatometry of the head. As a result, it was found that the Thévenin acoustic impedance is hardly influenced by the shape and size of the model head. The influence of head shape and size upon the maximum of absolute value of log magnitude and phase of Thévenin pressure was illustrated in terms of an equal magnitude contour and an equal phase contour. A final discussion deals with regards for an average head in shape and size as a head which is acoustically standard.
\end{abstract}

PACS number: 43. 88. Md, 43. 88. Vk, 43. 66. YW

\section{INTRODUCTION}

The dummyhead-headphone system, through which a listener is able to hear the same sound as he would hear in the sound field where the dummyhead is located, may play an important role in evaluation of room acoustics or acoustical devices for sound reproduction.

With the dummyhead-headphone system, it is necessary to determine a specification for a standard dummyhead so that the largest possible number of listeners hear with maximum authenticity. ${ }^{1)}$ To determine that specification, we need to evaluate the influence of the shape and size of the human head upon the sound reaching the ear. In evaluating the influence of the shape and size of human head, we adopted a two-step experimental scheme ${ }^{1)}$ : (1) determine the specification of head shape and size without the auricle, the nose and hair, and (2) ex-

$\dagger$ Now with SONY Corporation. amine the effect of attachment to the dummyhead with this specification.

Several dummyheads have been considered. For example, Mellert ${ }^{2)}$ constructed a dummyhead whose frequency characteristic was adapted to a desired curve of hearing sensitivity: The curve was obtained by averaging correlated structures of individual curves of hearing sensitivity of 17 subjects for lateral sound incidence. Burkhard and Sachs ${ }^{3)}$ designed a manikin "KEMAR" for hearing aid and related acoustic research: Dimensions of the torso and the head were based on published anthropometric data, and those of the auricles were based on data obtained for development of the KEMAR. They stated that the KEMAR represents a median individual in the human adult population, based on measurement of sound pressure at the earcanal entrance of 24 subjects for frequencies up to $10 \mathrm{kHz}$ with $90^{\circ}$ sound source azimuth angle.

Since the dummyhead-headphone system must reproduce the original sound field from any sound 
source, however, we should evaluate the influence of the shape and size of human head upon sound reaching the ear from the source in any direction.

Thévenin acoustic impedance and Thévenin pressure at the earcanal entrance were proposed for adoption as acoustical parameters for evaluating the effect of shape and size of individually different human heads. ${ }^{4)}$

The purpose of this paper is to evaluate numerically the influence of shape and size of the dummyhead upon Thévenin acoustic impedance and Thévenin pressure using two types of model heads, respectively a sphere and an oblate spheroid.

A rigid sphere whose circumference is equal to head circumference has already been used as a model of the human head in discussing the effect of intensity differences or time differences of pure tones at the ears in the binaural location. ${ }^{5}$ ) To get a higher approximation of the model head, furthermore, we deal with a rigid oblate spheroidal head whose ears are located at the opposite ends of the diameter on the axis of revolution of the spheroid.

The shape and size of the model head is changed according to head circumference and the cephalic index of somatometry of the human head. With the computational result of the normalized Thévenin pressure, we discuss whether an average head in shape and size can be regarded as a head which is acoustically standard.

\section{EQUATIONS FOR COMPUTING THÉVENIN ACOUSTIC IMPEDANCE AND THÉVENIN PRESSURE}

We begin with a brief explanation of Thévenin acoustic impedance and Thévenin pressure, and then consider the equations for their computation.

2.1 Thévenin Acoustic Impedance and Thévenin Pressure $^{6}$

Figure 1 shows an acoustic system representing a

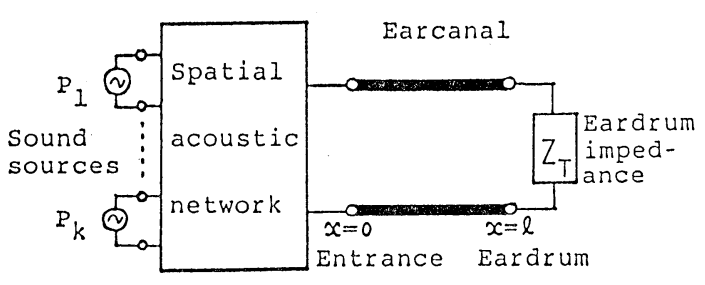

Fig. 1 Acoustic system representing a listener in the original sound field. listener in the sound field. The head, torso and auricles are included in the spatial acoustic network. Referring to the output terminals of the spatial acoustic network, the network can be replaced by its Thévenin equivalent. The result is shown in Fig. 2. The $P_{\mathrm{s}}(x=0)$ and $Z_{\mathrm{s}}(x=0)$ are the Thévenin pressure and the Thévenin acoustic impedance at the entrance of the earcanal, respectively. Note that Thévenin acoustic impedance corresponds to the acoustic radiation impedance of a virtual plane piston without mass at the entrance of the earcanal. And also note that Thévenin pressure corresponds to the sound pressure generated at the earcanal entrance when the earcanal is blocked by a rigid plug at its entrance.

To evaluate the influence of only the shape and size of the head upon the sound reaching the ear, let us consider a specific ear as shown in Fig. 3. The ear consists of a cylindrical concha embedded into the head and a cylindrical earcanal with a rigid termination.

Assuming that a plane wave of frequency $f$ propagates in the concha, we obtain the relation between the Thévenin acoustic impedance $z_{\mathrm{s}}(x=0)$ and the Thévenin pressure $p_{\mathrm{s}}(x=0)$ at the earcanal entrance and the Thévenin acoustic impedance $z_{\mathrm{s}}$ and the Thévenin pressure $p_{\mathrm{s}}$ at the concha entrance as shown in Eq. (1).

$$
\begin{aligned}
& p_{\mathrm{s}}(x=0)=p_{\mathrm{s}} /\left(\cos k d+j\left(z_{\mathrm{s}} / z_{\mathrm{c}}\right) \sin k d\right) \\
& z_{\mathrm{s}}(x=0)=z_{\mathrm{c}}\left(z_{\mathrm{s}}+j z_{\mathrm{c}} \tan k d\right) /\left(z_{\mathrm{c}}+j z_{\mathrm{s}} \tan k d\right)
\end{aligned}
$$

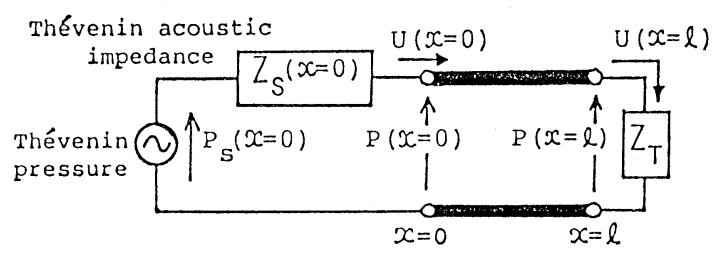

Fig. 2 Equivalent circuit of the system shown in Fig. 1.

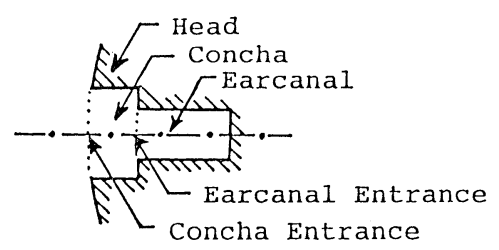

Fig. 3 Specific ear consisting of a cylindrical concha embedded into the head and a cylindrical earcanal. 


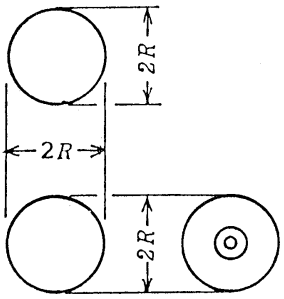

(a) Spherical head

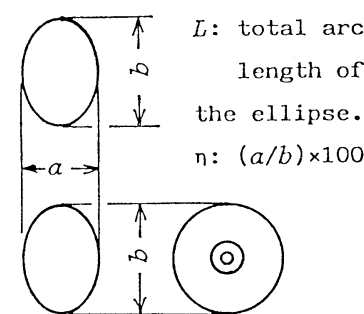

(b) Oblate spheroidal head
Fig. 4 Two types of model heads.

where $k=2 \pi f / c, z_{\mathrm{c}}=\rho c / S$, and $S$ is the cross-sectional area of the concha, $d$ the depth of the concha, $c$ the speed of sound in air, and $\rho$ the density of air.

The Thévenin acoustic impedance $z_{\mathrm{s}}$ and the Thévenin pressure $p_{\mathrm{s}}$ at the concha entrance of the model head can therefore be calculated in order to examine the influence of the shape and size of the head upon the sound reaching the ear. Figure 4 shows the two types of model heads: a rigid sphere, and a rigid oblate spheroid, each with ears located at the opposite ends of the axis of revolution.

Thévenin acoustic impedance and Thévenin pressure at the concha entrance of a spherical head can be calculated from the radiation impedance of a cap set of area $S$ in the spherical baffle and from the sound pressure on the surface of the sphere placed in the sound field, respectively. Those of the oblate spheroidal head can be calculated from the radiation impedance of a cap set of area $S$ in the oblate spheroidal baffle and from the sound pressure on the surface of the oblate spheroid placed in the sound field, respectively.

\subsection{Equations for Computing Thévenin Acoustic} Impedance and Thévenin Pressure at the Concha Entrance of a Spherical Head

The radiation impedance of a radially vibrating spherical cap set in a rigid spherical baffle is given by ${ }^{7)}$

$$
\begin{aligned}
z_{\mathrm{rad}, \mathrm{s}}= & \pi a^{2} \rho c \sum_{n=0}^{\infty} \frac{j}{2 n+1} \frac{k a h_{n}^{(2)}(k a)}{k a h_{n+1}^{(2)}(k a)-n h_{n}^{(2)}(k a)} \\
& \times\left\{P_{n-1}\left(\cos \theta_{0}\right)-P_{n+1}\left(\cos \theta_{0}\right)\right\}^{2}
\end{aligned}
$$

where the cap subtends an angle $2 \theta_{0}$ with the center of the sphere, and $a$ is the radius of the sphere, $h_{n}{ }^{(2)}$ the spherical Hankel function of the second kind, and $P_{n}$ the Legendre polynomial.

The Thévenin acoustic impedance at the concha entrance of a spherical head can then be calculated by

$$
z_{\mathrm{s}, \mathrm{s}}=z_{\mathrm{rad}, \mathrm{s}} / S^{2}
$$

where the concha has the same area $S$ as the cap:

$$
S=2 \pi a^{2}\left(1-\cos \theta_{0}\right) \text {. }
$$

The pressure at the surface of a scattering sphere which is placed at a distance $R$ from a point source with a simple harmonic volume flow $Q e^{j \omega t}$ is given by $^{8)}$

$$
p(\theta, R)=j \omega \rho \frac{Q e^{j \omega t}}{4 \pi a} \sum_{n=0}^{\infty}(2 n+1) \frac{h_{n}^{(2)}(k R) P_{n}(\cos \theta)}{k a h_{n+1}^{(2)}(k a)-n h_{n}^{(2)}(k a)}
$$

where $a$ is the radius of the sphere, and $\theta$ is the angle between the radius connecting the point on the surface of the sphere and the radius lying the direction of the point source.

The Thévenin pressure at the concha entrance of the spherical head can then be calculated by

$$
p_{\mathrm{s}, \mathrm{s}}(\theta, R)=p(\theta, R)
$$

2.3 Equations for Computing Thévenin Acoustic Impedance and Thévenin Pressure at the Concha Entrance of an Oblate Spheroidal Head

Let us consider a vibration of a cap set in a rigid oblate spheroidal baffle as shown in Fig. 5: The cap set, which is the part of the oblate spheroidal surface $u=u_{0}$ (const.) cut off by the co-axial cylinder with radius $a \cosh u_{0} \sin v_{0}$, vibrates like a piston with the constant velocity amplitude $V_{0}$ along the $z$-axis. The radiation impedance of this case is given by ${ }^{9)}$

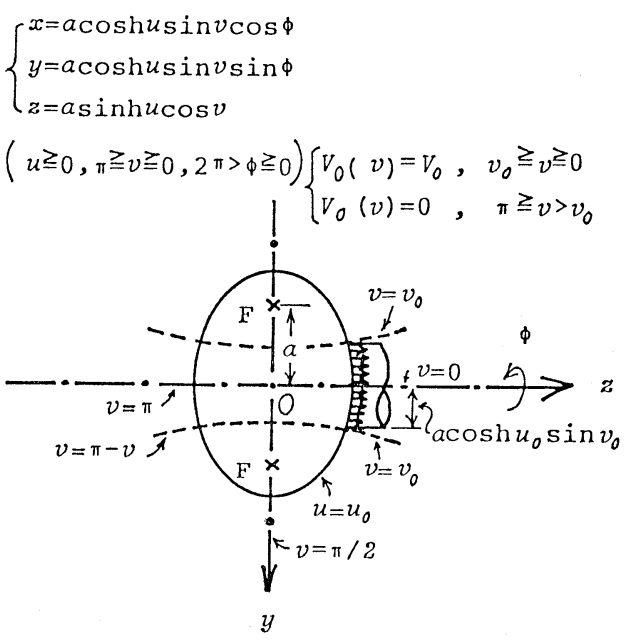

Fig. 5 Cap set in the oblate spheroidal baffle and coordinates. 


$$
\begin{aligned}
z_{\mathrm{rad}, \mathrm{os}}= & -\rho c k a 2 \pi a^{2} \cosh ^{2} u_{0} \sum_{n=0}^{\infty} \frac{\left\{\sigma_{n}^{0}\left(\eta_{0}\right)\right\}^{2}}{r e_{n}^{0^{\prime}}\left(-j k a, j \sinh u_{0}\right)} \\
& \times r e_{n}^{0}\left(-j k a, j \sinh u_{0}\right)
\end{aligned}
$$

where the $a$ is the semifocus distance,

$$
\begin{aligned}
& \eta_{0}=\cos v_{0} \\
& \sigma_{n}^{0}\left(\eta_{0}\right)=\left(1 / v_{0}\right) \int_{0}^{\pi} V_{0}(v) p e_{n}^{0}(-j k a, \cos v) \cos v \sin v d v \\
& N_{n, 0}=\int_{0}^{\pi}\left\{p e_{n}^{0}(-j k a, \cos v)\right\}^{2} \sin v d v
\end{aligned}
$$

and $p e_{n}^{0}$, and $r e_{n}^{0}$ are the spheroidal wave functions. ${ }^{10,11)}$

The Thévenin acoustic impedance at the concha entrance of the oblate spheroidal head can then be calculated by

$$
z_{\mathrm{s}, \mathrm{os}}=z_{\mathrm{rad}, \mathrm{os}} / S^{2}
$$

where the concha has the same area $S$ as the cap:

$$
\begin{aligned}
S= & \pi a^{2} \cosh u_{0}\left[\cosh u_{0}-\cos v_{0} \sqrt{\cos ^{2} v_{0}+\sinh ^{2} u_{0}}\right. \\
& +\sinh ^{2} u_{0} \log \left\{\left(1+\cosh u_{0}\right) /\left(\cos v_{0}\right.\right. \\
& \left.\left.+\sqrt{\left.\cos ^{2} v_{0}+\sinh ^{2} u_{0}\right)}\right\}\right] \quad\left(0<v_{0}<\pi / 2\right)
\end{aligned}
$$

Now let us consider the pressure at the surface of an oblate spheroid exposed to an incident plane wave. Figure 6 shows the angle $\theta$ of incidence, and the oblate spheroid whose rotational axis coincides with the $z$-axis. The pressure at the surface of the oblate spheroid, in the case of the rigid surface, is given by ${ }^{12)}$

$$
p(u, v, \phi)=j \omega \rho\left(\psi_{\mathrm{i}}+\psi_{\mathrm{s}}\right)
$$

where $\psi_{\mathrm{i}}$ and $\psi_{\mathrm{s}}$ are velocity potentials of the incident wave and the scattered wave, respectively and

$$
\begin{aligned}
\psi_{\mathrm{i}}= & A \sum_{n=0}^{\infty} \sum_{m=0}^{\infty} \epsilon_{m} j^{m} a_{n m} p e_{n}^{m}(-j k a, \cos \alpha) \\
& \times p e_{n}^{m}(-j k a, j \sinh u) p e_{n}^{m}(-j k a, \cos v) \cos m \phi
\end{aligned}
$$$$
\left(\epsilon_{0}=1, \epsilon_{1}=\epsilon_{2}=\ldots=2\right)
$$

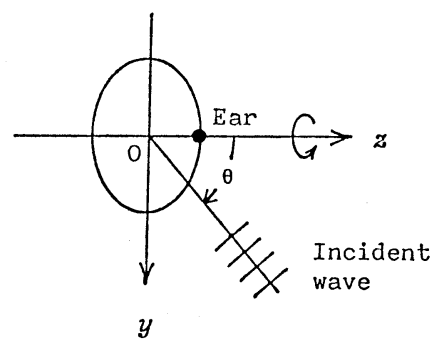

Fig. 6 Definition of the angle of incidence.

$$
\left\{\begin{array}{c}
\frac{k a \int_{0}^{\pi} J_{m}(k a \sin v) p e_{n}^{m}(-j k a, \cos v) \cos v \sin v d v}{\left\{p e_{n}^{m^{\prime}}(-j k a, 0)\right\}^{2} \int_{-1}^{1}\left\{p e_{n}^{m}(-j k a, x)\right\}^{2} d x} \\
\quad(n-m: \text { odd }) \\
\frac{\int_{0}^{\pi} J_{m}(k a \sin v) p e_{n}^{m}(-j k a, \cos v) \sin v d v}{\left\{p e_{n}^{m}(-j k a, 0)\right\}^{2} \int_{-1}^{1}\left\{p e_{n}^{m}(-j k a, x)\right\}^{2} d x} \\
(n-m: \text { even })
\end{array}\right.
$$$$
\psi_{\mathrm{s}}=A \sum_{n=0}^{\infty} \sum_{m=0}^{\infty} \epsilon_{m} j^{m} b_{n m} p e_{n}^{m}(-j k a, \cos \alpha)
$$$$
\times r e_{n}^{m}(-j k a, j \sinh u) p e_{n}^{m}(-j k a, \cos v) \cos m \phi
$$

$$
b_{n m}=-\frac{p e_{n}^{m^{\prime}}\left(-j k a, j \sinh u_{0}\right)}{r e_{n}^{m^{\prime}}\left(-j k a, j \sinh u_{0}\right)} a_{n m}
$$

Since the specific ear is located at one end of the diameter on the axis of revolution of the spheroid, we can put the coordinates of the ear, $(u, v, \phi)=$ $\left(u_{0}, 0,0\right)$ into Eqs. (9), (10), and (12). Using the formula

we obtain

$$
p e_{n}^{m}(-j k a, 1)=0 \text { for } m \neq 0,
$$

where

$$
p\left(u_{0}, 0,0\right)=j \omega \rho\left(\psi_{\mathrm{i}}+\psi_{\mathrm{s}}\right)
$$

$$
\begin{aligned}
\psi_{\mathrm{i}}= & A \sum_{n=0}^{\infty} a_{n_{0}} p e_{n}^{0}(-j k a, \cos \alpha) p e_{n}^{0}\left(-j k a, j \sinh u_{0}\right) \\
& \times p e_{n}^{0}(-j k a, 1) \\
\psi_{\mathrm{s}}= & A \sum_{n=0}^{\infty} b_{n_{0}} p e_{n}^{0}(-j k a, \cos \alpha) r e_{n}^{0}\left(-j k a, j \sinh u_{0}\right) \\
& \times p e_{n}^{0}(-j k a, 1)
\end{aligned}
$$$$
a_{n 0}
$$$$
=\left\{\begin{array}{c}
k a \int_{0}^{\pi} J_{0}(k a \sin v) p e_{n}^{0}(-j k a, \cos v) \cos v \sin v d v \\
\left\{p e_{n}^{0^{\prime}}(-j k a, 0)\right\}^{2} \int_{-1}^{1}\left\{p e_{n}^{0}(-j k a, x)\right\}^{2} d x \\
(n: \text { odd }) \\
\frac{\int_{0}^{\pi} J_{0}(k a \sin v) p e_{n}^{0}(-j k a, \cos v) \sin v d v}{\left\{p e_{n}^{0}(-j k a, 0)\right\}^{2} \int_{-1}^{1}\left\{p e_{n}^{0}(-j k a, x)\right\}^{2} d x} \\
(n: \text { even })
\end{array}\right.
$$

and 


\section{K. FUKUDOME and M. YAMADA: INFLUENCE OF THE SHAPE AND SIZE OF A DUMMYHEAD}

$$
b_{n 0}=-\frac{p e_{n}^{0^{\prime}}\left(-j k a, j \sinh u_{0}\right)}{r e_{n}^{0^{\prime}}\left(-j k a, j \sinh u_{0}\right)} a_{n 0}
$$

Since the integrals involved in Eq. (17) can be evaluated in terms of the series-representations, the coefficient $a_{n 0}$ is given by

$$
= \begin{cases}\frac{2 \sum_{s \geq-n / 2}^{\infty} A_{n, n+2 s}^{0} j^{n+2 s-1} j_{n+2 s}(k a) P_{n+2 s}(0)}{\left\{p e_{n}^{0^{\prime}}(-j k a, 0)\right\}^{2} N_{n 0}} & (n: \text { odd }) \\ \frac{2 \sum_{s \geq-n / 2}^{\infty} A_{n, n+2 s}^{0} j^{n+2 s} j_{n+2 s}(k a) P_{n+2 s}(0)}{\left\{p e_{n}^{0}(-j k a, 0)\right\}^{2} N_{n 0}} & (n: \text { even })\end{cases}
$$

where

$$
\begin{aligned}
N_{n 0} & =\int_{-1}^{1}\left\{p e_{n}^{0}(-j k a, x)\right\}^{2} d x \\
& =2 \sum_{s \geq-n / 2}^{\infty}\left(A_{n, n+2 s}^{0}\right)^{2} /\{2(n+2 s)+1\}
\end{aligned}
$$

and $A_{n, n+2 \mathrm{~s}}^{0}$ is the coefficient in the series-representation for $p e_{n}^{0}(-j k a, x)$ :

$$
p e_{n}^{0}(-j k a, x)=\sum_{s \geq-n / 2}^{\infty} A_{n, n+2 s}^{0} P_{n+2 s}(x)
$$

The Thévenin pressure at the concha entrance of the oblate spheroidal head can then be calculated by

$$
\begin{aligned}
p_{\mathrm{s}, \mathrm{os}}= & j \omega \rho A \sum_{n=0}^{\infty} a_{n 0}\left\{p e_{n}^{0}\left(-j k a, j \sinh u_{0}\right)\right. \\
& \left.-\frac{p e_{n}^{0^{\prime}}\left(-j k a, j \sinh u_{0}\right)}{r e_{n}^{0^{\prime}}\left(-j k a, j \sinh u_{0}\right)} r e_{n}^{0}\left(-j k a, \sinh u_{0}\right)\right\} \\
& \times p e_{n}^{0}(-j k a, \cos \alpha) p e_{n}^{0}(-j k a, 1)
\end{aligned}
$$

\section{MODEL HEADS FOR COMPUTATION}

To examine numerically the influence of the shape and size of the head upon Thévenin acoustic impedance and Thévenin pressure, we use two types of model heads shown in Fig. 4. The shape and size of the model head is determined by somatometry.

\subsection{Size of the Model Head for Computation of} Thévenin Acoustic Impedance

Three spherical heads and two oblate spheroidal heads are used.

The radii of the spherical heads are shown in Table 1. The dimensions ranged from the minimum to the maximum radius of a sphere corresponding to the human head in head length, head breadth, head
Table 1 Dimensions of the model head used in computing the Thévenin acoustic impedance.

Spherical heads

$(\mathrm{mm})$

\begin{tabular}{lccc}
\hline & \multicolumn{3}{c}{ Symbol } \\
\cline { 2 - 4 } & SH1 & SH2 & SH3 \\
\hline Radius & 60 & 100 & 140 \\
\hline Oblate spheroidal heads & \multicolumn{2}{c}{ Symbol } \\
\hline & \multicolumn{3}{c}{ OSH2 } \\
\cline { 2 - 3 } & OSH1 & 170 \\
\hline Minor diameter & 150 & 200 \\
Major diameter & 180 &
\end{tabular}

Table 2 Dimensions of the spherical model heads used in computing the Thévenin pressure.

$(\mathrm{mm})$

\begin{tabular}{lccc}
\hline & \multicolumn{3}{c}{ Symbol } \\
\cline { 2 - 4 } & SH1P & SH2P & SH3P \\
\hline Radius & 82.5 & 89.1 & 95.7 \\
Circumference & $\begin{array}{c}m-1.15 \sigma \\
(=518.3)\end{array}$ & $\begin{array}{c}m \\
(=559.6)\end{array}$ & $\begin{array}{c}m+1.15 \sigma \\
(=600.9)\end{array}$ \\
\hline
\end{tabular}

circumference, or face length based on the somatometrical data by Uchimura and Takeichi. ${ }^{13)}$

Also shown in Table 1 are the minor diameters and major diameters of the generating ellipses of the two oblate spheroidal heads.

\subsection{Size of the Model Head for Computation of Thévenin Pressure}

We first consider the model head whose size has one degree of freedom. Three spherical heads are used.

Dimensions of the model heads are shown in Table 2.

The circumferences of the spherical heads are equal to the average $m$ and the lower- and upper limits $m \pm 1.15 \sigma$ with respect to $75 \%$ distribution of the head circumference by Uchimura and Takeichi, ${ }^{13)}$ assuming that the distribution of head circumference is the normal distribution with average $m$ and variance $\sigma^{2}$.

Second, we consider the model head with two 
Table 3 The parameter values of nine oblate spheroidal heads used in computing the Thévenin pressure.

\begin{tabular}{cccc}
\hline \multirow{2}{*}{$\eta(\%)$} & \multicolumn{3}{c}{$L(\mathrm{~mm})$} \\
\cline { 2 - 4 } & $\bar{L}-1.15 \sigma_{L}=537.7$ & $\bar{L}=555.0$ & $\bar{L}+1.15 \sigma_{L}=572.2$ \\
\hline $\bar{\eta}-1.15 \sigma_{\eta}=79.13$ & $\mathrm{G}$ & $\mathrm{B}$ & $\mathrm{C}$ \\
$\bar{\eta}=83.57$ & $\mathrm{~F}$ & $\mathrm{~S}$ & $\mathrm{~A}$ \\
$\bar{\eta}+1.15 \sigma_{\eta}=88.01$ & $\mathrm{H}$ & $\mathrm{D}$ & $\mathrm{E}$ \\
\hline
\end{tabular}

degrees of freedom.

Nine oblate spheroidal heads are designated by the names of $\mathrm{S}$ and $\mathrm{A}$ to $\mathrm{H}$ as shown in Table 3 . The shape and size of the oblate spheroid is described by two parameters: respectively the minor to major diameter ratio $\eta$ and the total arc length $L$ of the generating ellipse of the spheroid. The parameters $\eta$ and $L$ correspond to the cephalic index I (ratio of head breadth to head length) and the head circumference respectively. Values used in Table 3 are based on the somatometrical data by Itou. ${ }^{14)}$ (Of his data, the average $\bar{\nu}$ and the standard deviation $\sigma_{\nu}$ of the cephalic index I and the head circumference of 807 young male Japanese adults ranging in age 21 to 23 are used: $\bar{\eta}=83.57, \sigma_{\eta}=3.86, \bar{L}=555.0(\mathrm{~mm})$ and $\sigma_{L}=15.0(\mathrm{~mm})$.)

\section{RESULTS AND DISCUSSION}

Computation of Thévenin acoustic impedance and Thévenin pressure of the model head was made up to the frequency $16 \mathrm{kHz}$.

4.1 The Influence of the Shape and Size of the Model Head upon Thévenin Acoustic Impedance

We calculated Eqs. (3) and (8) at a temperature of $20^{\circ} \mathrm{C}$ for the model heads in Table 1.

Figure 7 shows the Thévenin acoustic impedance at the concha entrance of the spherical head "SH2," being described in terms of magnitude and phase, $z_{\mathrm{s}, \mathrm{s}}=\left|z_{\mathrm{s}, \mathrm{s}}\right| \angle z_{\mathrm{s}, \mathrm{s}}$. Here a cylindrical concha of radius $11(\mathrm{~mm})$ was used in the specific ear.

Figure 8 shows the effect of the concha radius on the $z_{\mathrm{s}, \mathrm{os}}$ of the oblate spheroidal head "OSH1," being normalized by the $z_{\mathrm{s}, \mathrm{os}}$ in case of radius 11 $(\mathrm{mm})$. (Radii of the concha 9, 11, and $13(\mathrm{~mm})$ approximately correspond to the human conchae whose entrance area ${ }^{15}$ ) are minimum, average and maximum, respectively.)

In the following successive computation of
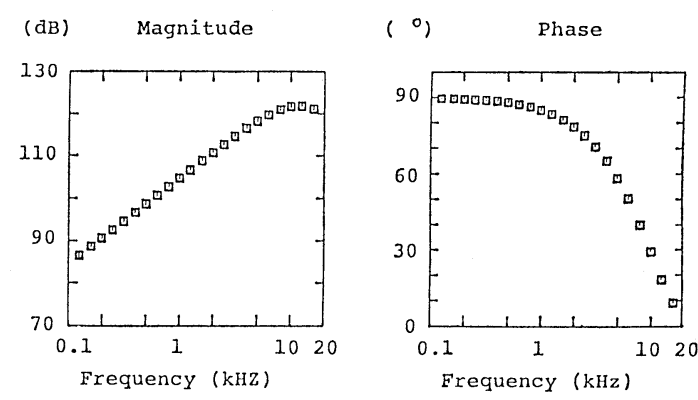

Fig. 7 Thévenin acoustic impedance at the concha entrance of the spherical head "SH2." The zero level in the magnitude characteristics is taken as 1 MKS acoustic ohm.
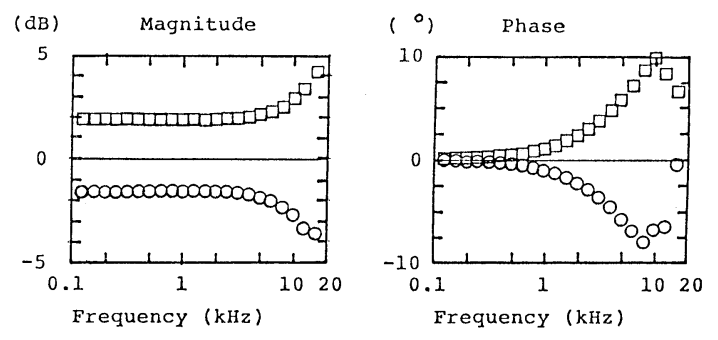

Fig. 8 Effect of the concha radius on the Thévenin acoustic impedance of the oblate spheroidal head "OSH1," being normalized by that of radius $11(\mathrm{~mm}) . \quad(\square 9(\mathrm{~mm})$ and $\bigcirc 13(\mathrm{~mm})$ ).

Thévenin acoustic impedance, a cylindrical concha of radius $11(\mathrm{~mm})$ is adopted giving an area of the concha entrance of $380\left(\mathrm{~mm}^{2}\right)$.

Figure 9 shows the ratio of Thévenin acoustic impedance $z_{\mathrm{s}, \mathrm{s}}$ or $z_{\mathrm{s}, \mathrm{os}}$ of the model head to that of the spherical head "SH2."

We can observe that the magnitude of the Thévenin acoustic impedance ratio is within $0.4 \mathrm{~dB}$, the phase of the ratio is within $2^{\circ}$, and the difference between 


\section{K. FUKUDOME and M. YAMADA: INFLUENCE OF THE SHAPE AND SIZE OF A DUMMYHEAD}
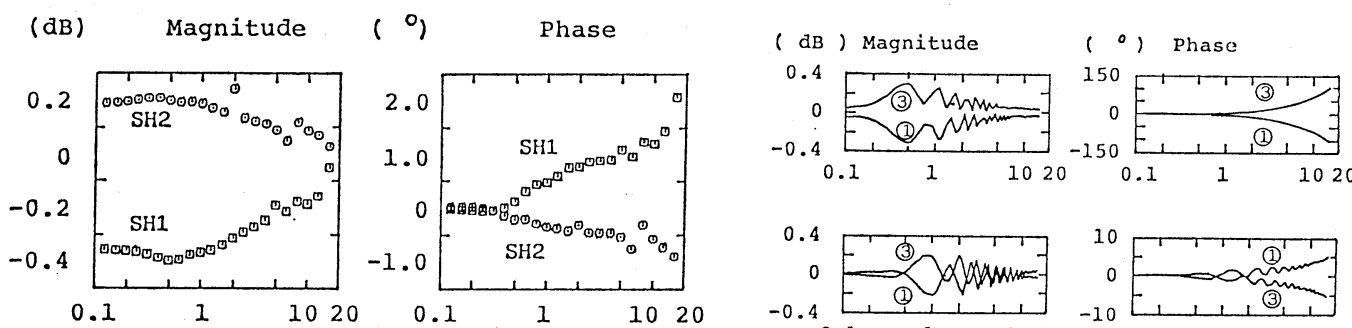

Angle of
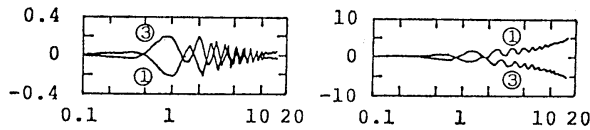

incidence

(a)
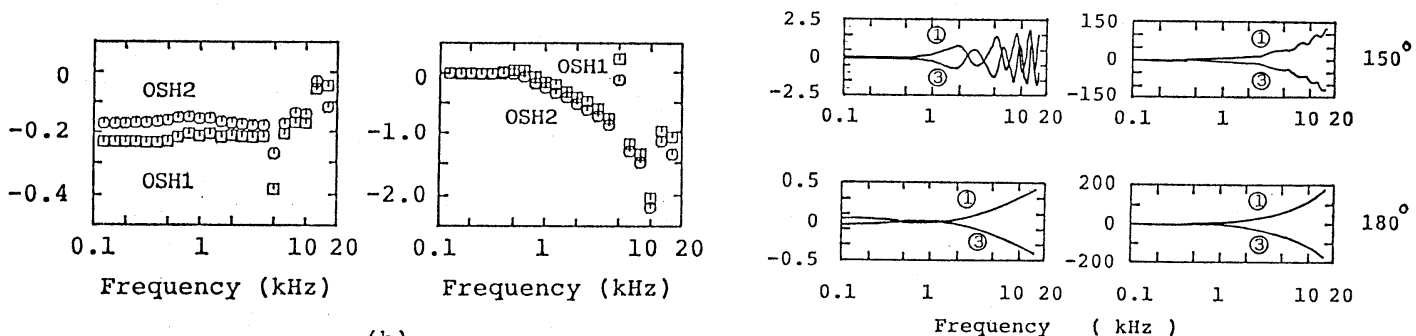

(b)

Fig. 9 Ratio of the Thévenin acoustic impedance of the model head in Table 1 to that of spherical head "SH2." (a) The $z_{\mathrm{s}, \mathrm{s}}$ of the spherical head, and (b) the $z_{\mathrm{s}, \mathrm{os}}$ of the oblate spheroidal head.

the two spheroidal heads is little. If the size of the head is described by its circumference, the spherical heads "SH1" and "SH3" are extraordinary heads because their circumferences are beyond the range of the circumference of human head. So, it can be seen that the Thévenin acoustic impedance is hardly influenced by the shape and size of the model head.

\subsection{The Influence of the Shape and Size of the} Model Head upon Thévenin Pressure

We calculated Eqs. (5) and (22) at a temperature of $20^{\circ} \mathrm{C}$ for the model heads in Tables 2 and 3.

We first examined the Thévenin pressure of the model head with one degree of freedom.

Figure 10 shows the Thévenin pressure ratio of the spherical head in Table 2 to that of the spherical head "SH2P." Here the distance from the point source $R$ is taken as $2(\mathrm{~m})$. The comparison shows that the log-magnitude and phase of the Thévenin pressure ratio of the "SH1P"-head is almost the same as those of the "SH3P"-head, where the two heads have an equal deviation from the average in circumference except for opposite signs.

Second, we examined the Thévenin pressure of the model head in Table 3 with two degrees of freedom.

Fig. 10 Ratio of the Thévenin pressure of the spherical head in Table 2 to that of spherical head "SH2P." (Curve with 1: the $p_{\mathrm{s}, \mathrm{s}}$ of head "SH1P." Curve with 3: the $p_{\mathrm{s}, \mathrm{s}}$ of head "SH3P.")
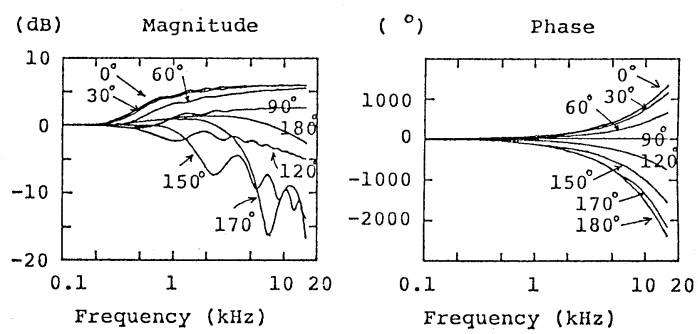

Fig. 11 Ratio of the Thévenin pressure of oblate spheroidal head " $S$ " to the pressure at its center before it is introduced into the plane wave field as a function of frequency with $\theta$ as a parameter.

Figure 11 shows the ratio of Thévenin pressure of the oblate spheroidal head " $S$ " to the pressure at its center before it is introduced into the plane wave field, as a function of frequency, with $\theta$ as a parameter. Further, Fig. 12 shows the magnitude frequency characteristics at additional angles of incidence to observe the behavior of Thévenin pressure in an irradiated region.

Before going into further results, let us define the normalized Thévenin pressure of the model head "X" by 

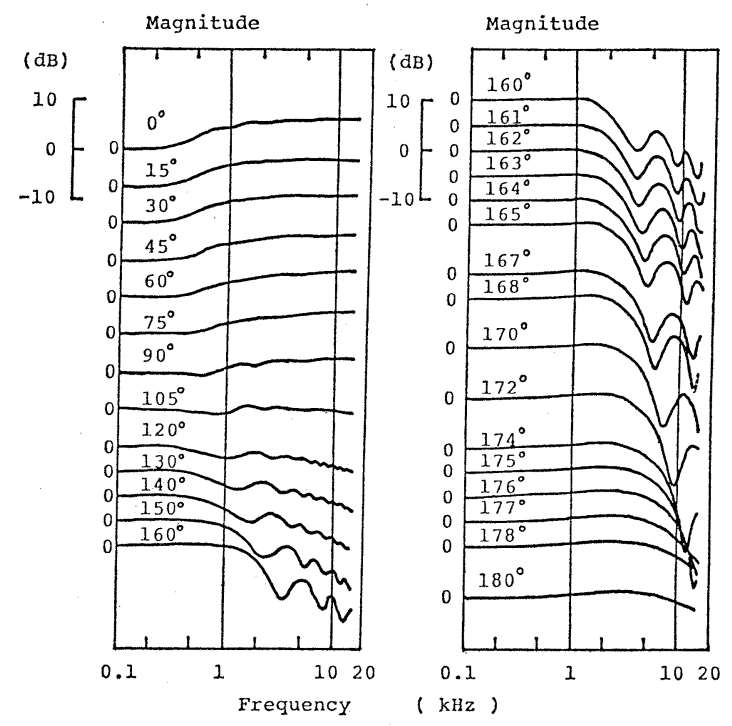

Fig. 12 Magnitude of the ratio in Fig. 11 at additional angles of incidence.

$$
\begin{aligned}
N T P_{\mathrm{x}}(\theta) & =\left|N T P_{\mathrm{x}}(\theta)\right| \angle N T P_{\mathrm{x}}(\theta) \\
& =\frac{\boldsymbol{P}_{\mathrm{s}, \mathrm{os}}(\theta) \text { of the head "X" }}{\boldsymbol{P}_{\mathrm{s}, \mathrm{os}}(\theta) \text { of the head "S" }}
\end{aligned}
$$

where the head " $\mathrm{X}$ " is any one in Table 3 .

Figure 13 compares normalized Thévenin pressure of the oblate spheroidal head " $B$ " with that of the oblate spheroidal head "D." These heads have the same value in parameter $L$. Their log magnitude in $\mathrm{dB}$ is almost the same except for opposite signs and their phases in degree are almost the same except for opposite signs, i.e.,

$$
N T P_{\mathrm{B}}(\theta) \fallingdotseq 1 / N T P_{\mathrm{D}}(\theta) \text {. }
$$

Figure 14 compares normalized Thévenin pressure of the oblate spheroidal head " $A$ " with that of the oblate spheroidal head "F." These heads have the same value in parameter $\eta$. We can observe that

$$
N T P_{\mathrm{A}}(\theta) \fallingdotseq 1 / N T P_{\mathrm{F}}(\theta)
$$

Figure 15 compares normalized Thévenin pressure of the oblate spheroidal head " $\mathrm{C}$ " with the value synthesized by the relationship,

$$
V S_{\mathrm{C}}(\theta)=N T P_{\mathrm{A}}(\theta) \cdot N T P_{\mathrm{B}}(\theta)
$$

Note that values of the parameters $L$ and $\eta$ of head "C" are $L(C)=\bar{L}+1.15 \sigma_{L}$ and $\eta(C)=\bar{\eta}-1.15 \sigma_{\eta}$ while those of head " $\mathrm{A}$ " and " $\mathrm{B}$ " are $L(A)=$ $\bar{L}+1.15 \sigma_{L}, \eta(A)=\bar{\eta}, L(B)=\bar{L}$, and $\eta(B)=\bar{\eta}-1.15 \sigma_{\eta}$,

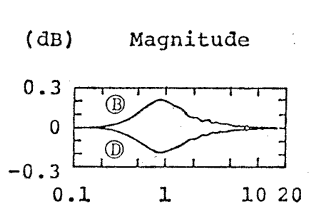

( $\left.{ }^{\circ}\right)$ Phase Angle of
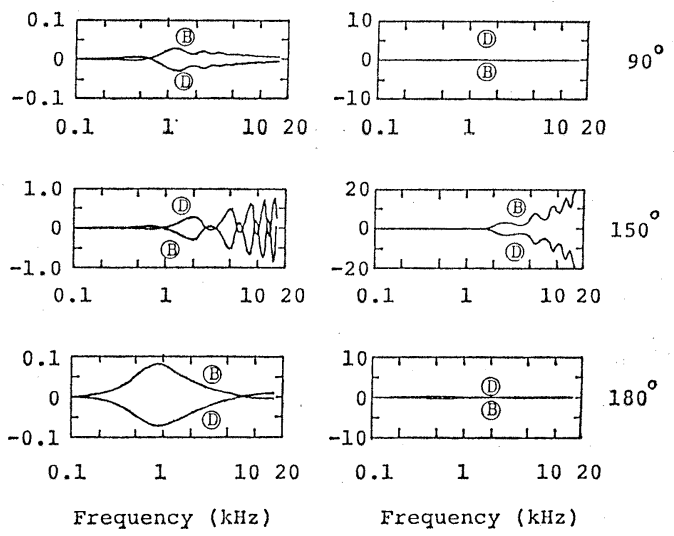

Fig. 13 Comparison of the normalized Thévenin pressure $N T P_{\mathrm{B}}(\theta)$ of oblate spheroidal head "B" with the $N T P_{\mathrm{D}}(\theta)$ of head "D."
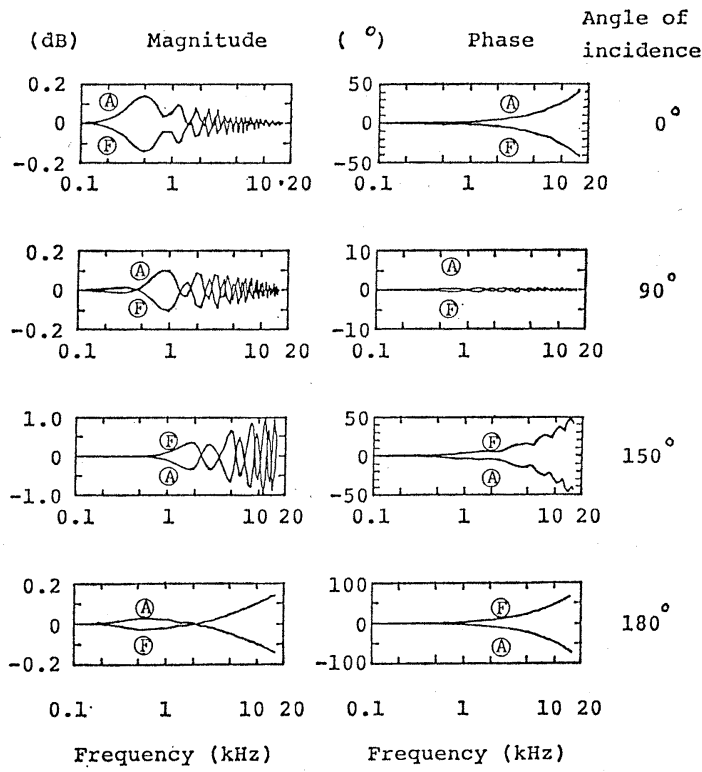

Fig. 14 Comparison of the $N T P_{\mathrm{A}}(\theta)$ with the $N T P_{F}(\theta)$.

respectively. We can observe that pairs of curves are almost the same in both log magnitude and phase, i.e., 


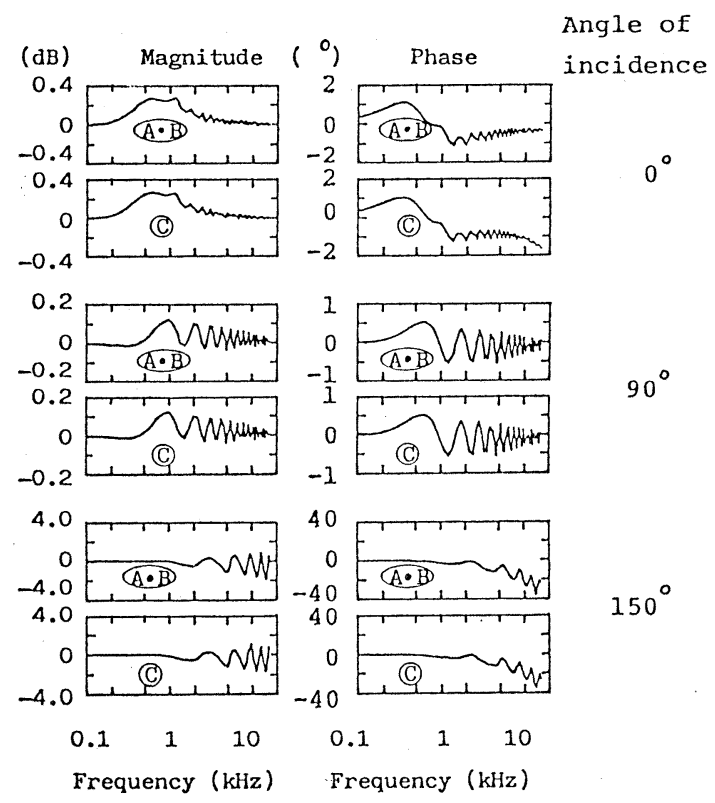

Fig. 15 Comparison of the $N T P_{\mathrm{C}}(\theta)$ with the value $V S_{\mathrm{C}}(\theta)$ synthesized by the relationship $V S_{\mathrm{C}}(\theta)=N T P_{\mathrm{A}}(\theta) N T P_{\mathrm{B}}(\theta)$.

$$
N T P_{\mathrm{C}}(\theta) \fallingdotseq N T P_{\mathrm{A}}(\theta) \cdot N T P_{\mathrm{D}}(\theta)
$$

Also, we can observe that there exist similar relationships as to the head "E," "G," or " $\mathrm{H}$," although figures are omitted here:

$$
\begin{aligned}
& N T P_{\mathrm{E}}(\theta) \doteqdot N T P_{\mathrm{A}}(\theta) \cdot N T P_{\mathrm{D}}(\theta) \\
& N T P_{\mathrm{G}}(\theta) \doteqdot N T P_{\mathrm{F}}(\theta) \cdot N T P_{\mathrm{B}}(\theta)
\end{aligned}
$$

and,

$$
N T P_{\mathrm{H}}(\theta) \fallingdotseq N T P_{\mathrm{F}}(\theta) \cdot N T P_{\mathrm{D}}(\theta)
$$

Now, let us discuss how the $\log$ magnitude of normalized Thévenin pressure, $20 \cdot \log _{10}\left|N T P_{\mathrm{X}}(\theta)\right|$, behaves as a function of frequency. After computations for further values of angle $\theta$, whose figures are omitted here, the following became clear:

(1) For values of $\theta, 0^{\circ} \leqq \theta \leqq 90^{\circ}$, the log magnitude of normalized Thévenin pressure has a maximum deviation at a frequency less than $2 \mathrm{kHz}$, and its absolute value is within $\pm 0.3 \mathrm{~dB}$.

(2) For values of $\theta, 90^{\circ}<\theta<176^{\circ}$, the $\log$ magnitude does not exceed $\pm 0.7 \mathrm{~dB}$ up to the frequency $2 \mathrm{kHz}$, and its envelope increases monotonically along with the axis of frequency higher than $2 \mathrm{kHz}$. Furthermore, the extremal frequencies, where the log magnitude has extremal values, rise as the angle $\theta$ is increased.

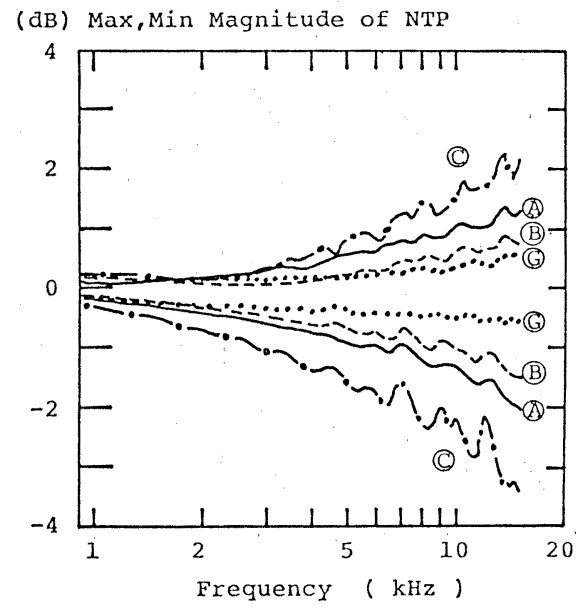

(a)

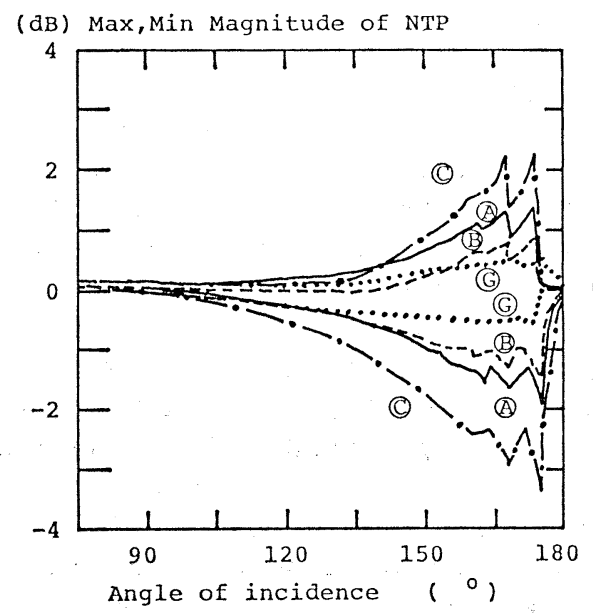

Fig. 16 Maximum and minimum log magnitude of the normalized Thévenin pressure: (a) when the angle $\theta$ is changed from $0^{\circ}$ to $180^{\circ}$ at each frequency, and (b) when the frequency is changed from 0.1 $\mathrm{kHz}$ to $15 \mathrm{kHz}$ at each angle.

(3) For values of $\theta \geqq 176^{\circ}$, the log magnitude does not exceed $\pm 0.1 \mathrm{~dB}$ for frequency less than $2 \mathrm{kHz}$ and the extremal value of the $\log$ magnitude does not appear up to the frequency $16 \mathrm{kHz}$.

Figure 16 shows the maximum and minimum log magnitude of normalized Thévenin pressure. The upper figure (a) shows the maximum and minimum when the angle of incidence is changed from $0^{\circ}$ to $180^{\circ}$ at each frequency. The lower figure (b) shows the maximum and minimum when the frequency is changed from $0.1 \mathrm{kHz}$ to $15 \mathrm{kHz}$ at each angle of incidence. (Angles and frequencies used in the 


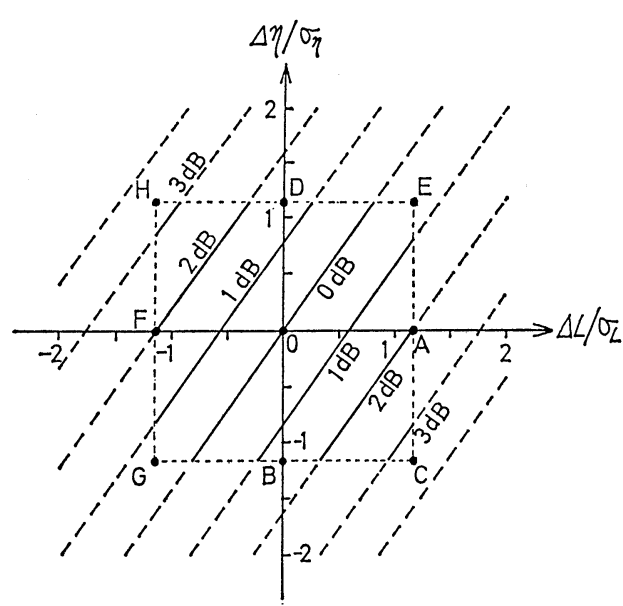

Fig. 17 Equal magnitude contour of the maximum of absolute value of the $\log$ magnitude of the Thévenin pressure.

computation are shown in Table A in the Appendix.)

In the figure, those values of heads " $F$," "D," "H," and "E" are omitted because they are nearly equal to the negative values of the log magnitude of heads "A," "B," "C," and "G," respectively.

At the highest frequency of concern, an envelope value of each curve in Fig. 16(a), gives the maximum deviation of the log magnitude of Thévenin pressure of the head " $X$ " from that of the head "S."

We can then determine the influence of shape and size of the oblate spheroidal head upon the maximum absolute value of the log magnitude of Thévenin pressure in terms of the equal magnitude contour in the $\Delta L / \sigma_{L}-\Delta \eta / \sigma_{\eta}$ plane as shown in Fig. 17 (where $\Delta L=L(X)-\bar{L}$, and $\Delta \eta=\eta(X)-\bar{\eta}$ ). Here, the frequency up to $15 \mathrm{kHz}$ is concerned, and customary interpolation which is used in reading between the lines of a published table is utilized, and a relationship similar to the one Eqs. (26) (29),

$$
N T P_{\mathrm{T}}(\theta) \doteqdot N T P_{\mathrm{v}}(\theta) N T P_{\mathrm{v}}(\theta)
$$

is used. $\quad N T P_{\mathrm{T}}(\theta)$ denotes the normalized Thévenin pressure of an arbitrary oblate spheroidal head "T," and $N T P_{\mathrm{v}}(\theta)$ and $N T P_{\mathrm{V}}(\theta)$ denote the ones of the head " $U$ " and " $V$ " associated with the head "T." Their parameters are $L(T)=\bar{L}+x \sigma_{L}, \quad \eta(T)=$ $\bar{\eta}+y \sigma_{\eta}, L(U)=\bar{L}+x \sigma_{x}, \eta(U)=\bar{\eta}, L(V)=\bar{L}, \eta(V)$ $=\bar{\eta}+y \sigma_{\eta}$, and $0<|x|,|y|<1.15$. Also shown in Fig. 18 are the maxima associated with heads " $A$ " to "H."

Next, let us discuss how the phase of normalized
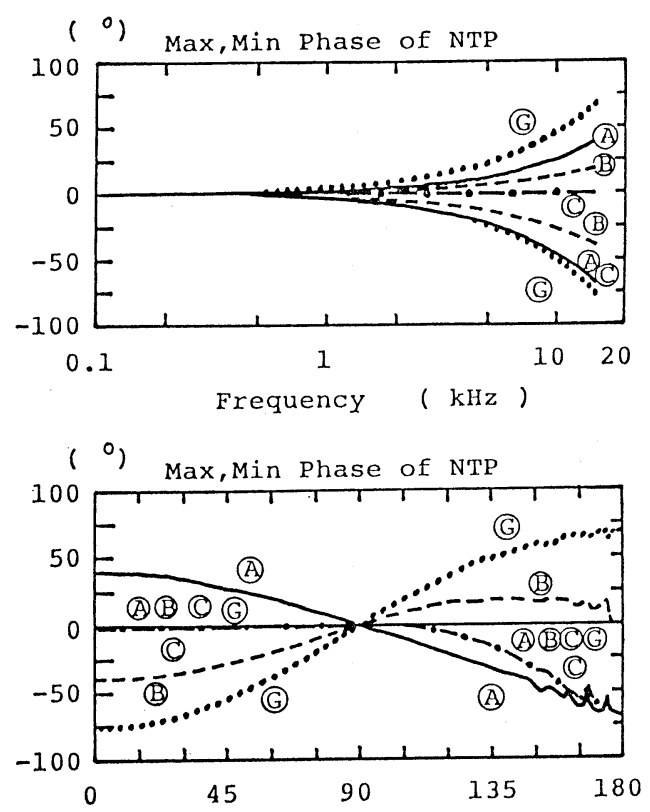

Angle of incidence ( ${ }^{\circ}$ )

Fig. 18 Maximum and minimum phase of the normalized Thévenin pressure: (a) when the angle $\theta$ is changed from $0^{\circ}$ to $180^{\circ}$ at each frequency, and (b) when the frequency is changed from $0.1 \mathrm{kHz}$ to $15 \mathrm{kHz}$ at each angle.

Thévenin pressure, $\angle N T P_{\mathrm{x}}(\theta)$, behaves as a function of frequency.

Figure 18 shows the maximum and minimum phase of normalized Thévenin pressure. The upper figure (a) shows the maximum and minimum phase when the angle of incidence is changed from $0^{\circ}$ to $180^{\circ}$ at each frequency. The lower figure (b) shows the maximum and mimumum phase when the frequency is changed from $0.1 \mathrm{kHz}$ to $15 \mathrm{kHz}$ at each angle of incidence. Note that those phases of heads "F," " $D$," "H," and "E" are nearly equal to the negative values of those phases of heads "A," "B," "C," and "G," respectively.

It can be seen that the maximum absolute value of the phase of normalized Thévenin pressure occurs at the highest frequency of concern and at the angle of incidence $\theta=0^{\circ}$, or $\theta=180^{\circ}$.

This maximum gives maximum deviation of the phase of Thévenin pressure of head " $X$ " from that of head "S." By setting the highest frequency of concern as $15 \mathrm{kHz}$, and using customary interpolation and the relationship Eq. (30), we can determine 


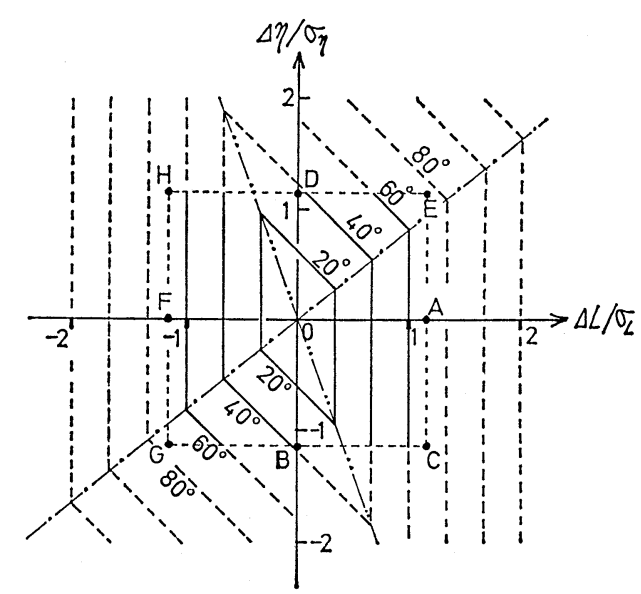

Fig. 19 Equal phase contour of the maximum of absolute value of the phase of the Thévenin pressure.

the influence of shape and size of the oblate spheroidal head upon the maximum absolute value of the phase of Thévenin pressure in terms of the equal phase contour in the $\Delta L / \sigma_{L}-\Delta \eta / \sigma_{\eta}$ plane as shown in Fig. 19. Also shown in Fig. 19 are the maxima associated with the heads "A" to " $\mathrm{H}$."

\subsection{Discussion of a Standard Dummyhead}

Let us discuss whether an average head in shape and size can be regarded as a head which is acoustically standard. Here, let a dummyhead's definition of acoustically standard be the following: (1) to be typical and (2) to have the smallest possible difference between Thévenin pressure of the dummyhead and that of a head chosen arbitrarily from the head population. Note that listener's eardrum sound pressure reproduced through a dummyheadheadphone system differs by a factor $C_{\mathrm{ps}}$ from that of the same listener in the original sound field ${ }^{6)}$; a factor $C_{\mathrm{ps}}$ denotes the ratio of the Thévenin pressure of the listener's head to that of the dummyhead.

In Sect. 4.2, we examined the influence of the shape and size of the oblate spheroidal model head upon Thévenin pressure in terms of both logmagnitude and phase of NTP. Head " $\mathrm{S}$ " possessing the average parameters, $\bar{\eta}$ and $\bar{L}$, had the average value of NTP for heads whose parameter values $\eta$ and $L$ lie within the intervals $\bar{\eta} \pm 1.15 \sigma_{\eta}$ and $\bar{L} \pm 1.15 \sigma_{L}$, respectively. (Note that in a normal distribution, about 75 percent of all heads must lie within this interval). Also, head " $\mathrm{S}$ " is typical because it is representative of the oblate spheroidal heads with two degrees of freedom. We can therefore regard head " $S$ " as an acoustically standard dummyhead. This suggests that a similar result may be expected in the case of the model head whose shape and size are described by many degrees of freedom. Therefore, if we determine a specification of dummyhead according to average values obtained by three-dimensional measurement of human head, ${ }^{1)}$ the dummyhead might be regarded as a head which is acoustically standard.

Up to this point, we have discussed the standard dummyhead on the basis of monaural attributes of ear input sound signals. Further we should discuss this problem on the basis of interaural attributes of ear input sound signals to clarify an equalization method of reproducing a three-dimensional sound field for unspecified listeners through dummyheadheadphone systems. This is dealt with elsewhere, but the oblate spheroidal head " $S$ " can serve for numerical evaluation of the validity of that equalization method.

\section{CONCLUSION}

To evaluate the influence of the shape and size of human head upon sound reaching the ear, we have examined the influence of the shape and size of the dummyhead upon Thévenin acoustic impedance and Thévenin pressure using two types of model heads, i.e., spherical and oblate spheroidal heads. Since the oblate spheroidal head has two degrees of freedom, two parameters 1) the minor to major diameter ratio $\eta$ and 2) the total arc length $L$, of the generating ellipse of the spheroid, have been adopted as parameters describing the shape and size of the head. The ranges of values of the two parameters have been determined according to somatometrical data of the cephalic index, defined by the ratio of head breadth to head length, and the head circumference, respectively.

The result we have observed shows that the Thévenin acoustic impedance is hardly influenced by shape and size of the model head, and Thévenin pressure in the shadow region is considerably influenced at high frequencies by the shape and size. We have determined the influence of shape and size of an oblate spheroidal head upon the maximum of normalized Thévenin pressure in terms of the equal magnitude contour and equal phase contour in the $\Delta L-\Delta \eta$ plane. Further we have discussed 
whether an average head in shape and size could be regarded as a head which is acoustically standard.

The problems which remain are to examine the influence of shape and size of the dummyhead upon (1) interaural differences in terms of the ratio of normalized Thévenin pressure at the left ear to that at the right ear, and (2) in the case of the different locations of the two ears whose angular separation on the horizontal head contour is not equal to $180^{\circ}$.

\section{ACKNOWLEDGEMENTS}

The authors would like to express their appreciation to Emeritus Prof. Yasuo Makita of Kyushu Institute of design and Prof. Hikaru Date (formerly of Kyushu Institute of Design, now with Yamagata University) for their sustained guidance and encouragement.

\section{REFERENCES}

1) K. Fukudome, "A three-dimensional measurement of human head-For the purpose of dummyhead construction," J. Acoust. Soc. Jpn. (E) 4, 35-43 (1983).

2) V. Mellert, "Construction of a dummy head after new measurements of thresholds of hearing," J. Acoust. Soc. Am. 51, 1359-1361 (1972).

3) M. D. Burkhard and R. M. Sachs, "Anthropometric manikin for acoustic research," J. Acoust. Soc. Am. 58, 214-222 (1975).

4) K. Fukudome, "The Thévenin acoustic impedance and pressure of dummyheads," Proc. 10th Int. Congr. Acoust. (Sydney) 3, L-13.2 (1980).

5) For example, G. W. Stewart, "The function of intensity and phase in the binaural location of pure tones. I.," Phys. Rev. (2nd Ser.) XV, 425-431 (1920).

6) K. Fukudome, "Equalization for the dummy-headheadphone system capable of reproducing true directional information," J. Acoust. Soc. Jpn. (E)
1, 59-67 (1980).

7) T. Hayasaka and S. Yoshikawa, Theory of MechanoAcoustic Vibration (Maruzen, Tokyo, 1974), p. 635 (in Japanese).

8) T. Hayasaka and S. Yoshikawa, Theory of MechanoAcoustic Vibration (Maruzen, Tokyo, 1974), p. 684 (in Japanese).

9) T. Nimura and Y. Watanabe, "Sound radiation from a circular disc of one side being vibrated like a piston while the other remaining still," J. Inst. Electr. Commun. Eng. Jpn. 33, 300-304 (1950) (in Japanese).

10) M. Kotani, "An acoustical problem relating to the theory of Rayleigh disc," Proc. Phys. Math. Soc. Jpn. 15, 30-57 (1933).

11) T. Nimura and Y. Watanabe, "Spheroidal wave functions and tables for them, I \& II," Rec. E. C. E. C. Tohoku Univ. $18(16,17)(1949)$ (in Japanese).

12) K. Ikegaya, "Characteristics of the sphere and circular disk in the sound field as the models of acoustical devices," Electr. Commun. Lab. Tech. J. 7, 127-188 (1958) (in Japanese).

13) Y. Uchimura and K. Takeichi, "Anthropometric survey of human figure measurement of head and face," Bull. Ind. Prod. Res. Inst. 62, 57-64 (1970) (in Japanese).

14) M. Itou, "A somatometrical study on the shape of adult head," St. Marianna Kenkyujo Gyohou 7, 1-172 (1954) (in Japanese).

15) K. Fukudome, M. Matsumoto, and K. Nikaido, "Somatometry of the human auricle for dummyhead construction-A photogrammetric study of moulded plaster auricles made from impressions of the human left ears," The Autumn Meet. Acoust. Soc. Jpn., 299-300 (1983) (in Japanese).

\section{APPENDIX}

In the computation of Thévenin pressure, frequencies and angles of incidence in Table $\mathrm{A}$ are used. Note that curves in the figures associated with Thévenin pressure are drawn in continuous form.

Table A Frequencies and angles of incidence used in the computation of the Thévenin pressure.

(a) Frequencies $(\mathrm{Hz})$

\begin{tabular}{rrrrrrrr}
\hline 100 & 125 & 160 & 200 & 250 & 315 & 400 & 500 \\
630 & 800 & 1,000 & 1,150 & 1,250 & 1,400 & 1,600 & 1,800 \\
2,000 & 2,250 & 2,500 & 2,750 & 2,950 & 3,150 & 3,400 & 3,700 \\
4,000 & 4,350 & 4,700 & 5,000 & 5,350 & 5,700 & 6,000 & 6,300 \\
6,600 & 6,900 & 7,200 & 7,600 & 8,000 & 8,400 & 8,800 & 9,200 \\
9,600 & 10,000 & 10,500 & 11,000 & 11,500 & 12,000 & 12,500 & 13,000 \\
13,500 & 14,000 & 14,500 & 15,000 & 15,500 & 16,000 & & \\
\hline
\end{tabular}

(b) Angles of incidence $\left({ }^{\circ}\right)$

\begin{tabular}{cccccccc}
\hline 0 & 15 & 30 & 45 & 60 & 75 & 90 & 105 \\
120 & 130 & 135 & 140 & 150 & 155 & 160 & $161^{*}$ \\
$162^{*}$ & $163^{*}$ & 164 & 165 & $167^{*}$ & 168 & 170 & 172 \\
174 & 175 & 176 & $177^{*}$ & $178^{*}$ & 180 & & \\
\hline
\end{tabular}

Note: Figures accompanied by * are used in the computation for some model heads. 